\title{
Mittens: An Extension of GloVe for Learning Domain-Specialized Representations
}

\author{
Nicholas Dingwall \\ Roam Analytics \\ nickeroamanalytics.com
}

\author{
Christopher Potts \\ Stanford University and Roam Analytics \\ cgpottsastanford.edu
}

\begin{abstract}
We present a simple extension of the GloVe representation learning model that begins with general-purpose representations and updates them based on data from a specialized domain. We show that the resulting representations can lead to faster learning and better results on a variety of tasks.
\end{abstract}

\section{Introduction}

Many NLP tasks have benefitted from the public availability of general-purpose vector representations of words trained on enormous datasets, such as those released by the GloVe (Pennington et al., 2014) and fastText (Bojanowski et al., 2016) teams. These representations, when used as model inputs, have been shown to lead to faster learning and better results in a wide variety of settings (Erhan et al., 2009, 2010; Cases et al., 2017).

However, many domains require more specialized representations but lack sufficient data to train them from scratch. We address this problem with a simple extension of the GloVe model (Pennington et al., 2014) that synthesizes general-purpose representations with specialized data sets. The guiding idea comes from the retrofitting work of Faruqui et al. (2015), which updates a space of existing representations with new information from a knowledge graph while also staying faithful to the original space (see also Yu and Dredze 2014; Mrkšić et al. 2016; Pilehvar and Collier 2016). We show that the GloVe objective is amenable to a similar retrofitting extension. We call the resulting model 'Mittens', evoking the idea that it is 'GloVe with a warm start' or a 'warmer GloVe'.

Our hypothesis is that Mittens representations synthesize the specialized data and the generalpurpose pretrained representations in a way that gives us the best of both. To test this, we conducted a diverse set of experiments. In the first, we learn GloVe and Mittens representations on IMDB movie reviews and test them on separate IMDB reviews using simple classifiers. In the second, we learn our representations from clinical text and apply them to a sequence labeling task using recurrent neural networks, and to edge detection using simple classifiers. These experiments support our hypothesis about Mittens representations and help identify where they are most useful.

\section{Mittens}

This section defines the Mittens objective. We first vectorize GloVe to help reveal why it can be extended into a retrofitting model.

\subsection{Vectorizing GloVe}

For a word $i$ from vocabulary $V$ occurring in the context of word $j$, GloVe learns representations $w_{i}$ and $\widetilde{w}_{j}$ whose inner product approximates the logarithm of the probability of the words' cooccurrence. Bias terms $b_{i}$ and $\tilde{b}_{j}$ absorb the overall occurrences of $i$ and $j$. A weighting function $f$ is applied to emphasize word pairs that occur frequently and reduce the impact of noisy, low frequency pairs. This results in the objective

$$
J=\sum_{i, j=1}^{V} f\left(X_{i j}\right)\left(w_{i}^{\top} \widetilde{w}_{j}+b_{i}+\tilde{b}_{j}-\log X_{i j}\right)^{2}
$$

where $X_{i j}$ is the co-occurrence of $i$ and $j$. Since $\log X_{i j}$ is only defined for $X_{i j}>0$, the sum excludes zero-count word pairs. As a result, existing implementations of GloVe use an inner loop to compute this cost and associated derivatives.

However, since $f(0)=0$, the second bracket is irrelevant whenever $X_{i j}=0$, and so replacing $\log X_{i j}$ with

$$
g\left(X_{i j}\right)= \begin{cases}k, & \text { for } X_{i j}=0 \\ \log \left(X_{i j}\right), & \text { otherwise }\end{cases}
$$




\begin{tabular}{lrrrrrr}
\hline & \multicolumn{9}{c}{ Vocabulary size } \\
Implementation & $5 \mathrm{~K}$ & $10 \mathrm{~K}$ & $20 \mathrm{~K}$ & $5 \mathrm{~K}$ & $10 \mathrm{~K}$ & $20 \mathrm{~K}$ \\
\hline Non-vectorized TensorFlow & 14.02 & 63.80 & 252.65 & 13.56 & 55.51 & 226.41 \\
Vectorized Numpy & 1.48 & 7.35 & 50.03 & - & - & - \\
Vectorized TensorFlow & 1.19 & 5.00 & 28.69 & 0.27 & 0.95 & 3.68 \\
Official GloVe (in C) & 0.66 & 1.24 & 3.50 & - & - & - \\
\hline
\end{tabular}

Table 1: Speed comparisons. The values are seconds per iteration, averaged over 10 iterations each on 5 simulated corpora that produced count matrices with about $10 \%$ non-zero cells. Only the training step for each model is timed. The CPU experiments were done on a machine with a $3.1 \mathrm{GHz}$ Intel Core 17 chip and $16 \mathrm{~GB}$ of memory, and the GPU experiments were done on machine with a 16 GB NVIDIA Tesla V100 GPU and 61 GB of memory. Dashes mark tests that aren't applicable because the implementation doesn't perform GPU computations.

(for any $k$ ) does not affect the objective and reveals that the cost function can be readily vectorized as

$$
J=f(X) M^{\top} M
$$

where $M=W^{\top} \widetilde{W}+b 1^{\top}+1 \tilde{b}^{\top}-g(X) . W$ and $\widetilde{W}$ are matrices whose columns comprise the word and context embedding vectors, and $g$ is applied elementwise. Because $f\left(X_{i j}\right)$ is a factor of all terms of the derivatives, the gradients are identical to the original GloVe implementation too.

To assess the practical value of vectorizing GloVe, we implemented the model ${ }^{1}$ in pure Python/Numpy (van der Walt et al., 2011) and in TensorFlow (Abadi et al., 2015), and we compared these implementations to a non-vectorized TensorFlow implementation and to the official GloVe C implementation (Pennington et al., 2014). ${ }^{2}$ The results of these tests are in tab. 1. Though the $\mathrm{C}$ implementation is the fastest (and scales to massive vocabularies), our vectorized TensorFlow implementation is a strong second-place finisher, especially where GPU computations are possible.

\subsection{The Mittens Objective Function}

This vectorized implementation makes it apparent that we can extend GloVe into a retrofitting model by adding a term to the objective that penalizes the squared euclidean distance from the learned embedding $\widehat{w}_{i}=w_{i}+\widetilde{w}_{i}$ to an existing one, $r_{i}$ :

$$
J_{\text {Mittens }}=J+\mu \sum_{i \in R}\left\|\widehat{w}_{i}-r_{i}\right\|^{2} .
$$

\footnotetext{
${ }^{1}$ https://github.com/roamanalytics/mittens

${ }^{2}$ We also considered a non-vectorized Numpy implementation, but it was too slow to be included in our tests (a single iteration with a $5 \mathrm{~K}$ vocabulary took $2 \mathrm{hrs} 38 \mathrm{mins}$ ).
}

Here, $R$ contains the subset of words in the new vocabulary for which prior embeddings are available (i.e., $R=V \cap V^{\prime}$ where $V^{\prime}$ is the vocabulary used to generate the prior embeddings), and $\mu$ is a non-negative real-valued weight. When $\mu=0$ or $R$ is empty (i.e., there is no original embedding), the objective reduces to GloVe's.

As in retrofitting, this objective encodes two opposing pressures: the GloVe objective (left term), which favors changing representations, and the distance measure (right term), which favors remaining true to the original inputs. We can control this trade off by decreasing or increasing $\mu$.

In our experiments, we always begin with 50-dimensional 'Wikipedia 2014 + Gigaword 5' GloVe representations ${ }^{3}$ - henceforth 'External GloVe' - but the model is compatible with any kind of "warm start".

\subsection{Notes on Mittens Representations}

GloVe's objective is that the log probability of words $i$ and $j$ co-occurring be proportional to the dot product of their learned vectors. One might worry that Mittens distorts this, thereby diminishing the effectiveness of GloVe. To assess this, we simulated 500-dimensional square count matrices and original embeddings for $50 \%$ of the words. Then we ran Mittens with a range of values of $\mu$. The results for five trials are summarized in fig. 1: for reasonable values of $\mu$, the desired correlation remains high (fig. 1a), even as vectors with initial embeddings stay close to those inputs, as desired (fig. 1b).

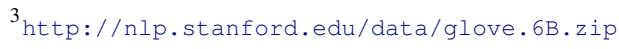




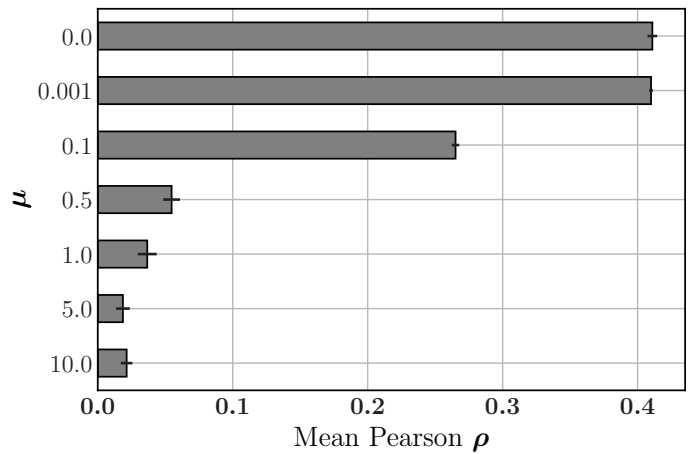

(a) Correlations between the dot product of pairs of learned vectors and their $\log$ probabilities.

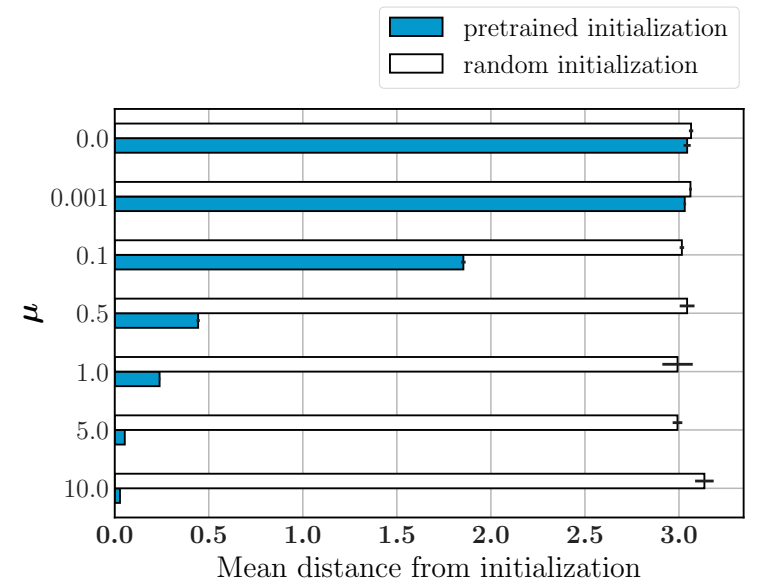

(b) Distances between initial and learned embeddings, for words with and without pretrained initializations. As $\mu$ gets larger, the pressure to stay close to the original increases.

Figure 1: Simulations assessing Mittens' faithfulness to the original GloVe objective and to its input embeddings. $\mu=0$ is regular GloVe.

\section{Sentiment Experiments}

For our sentiment experiments, we train our representations on the unlabeled part of the IMDB review dataset released by Maas et al. (2011). This simulates a common use-case: Mittens should enable us to achieve specialized representations for these reviews while benefiting from the large datasets used to train External GloVe.

\subsection{Word Representations}

All our representations begin from a common count matrix obtained by tokenizing the unlabeled movie reviews in a way that splits out punctuation, downcases words unless they are written in all uppercase, and preserves emoticons and other common social media mark-up. We say word $i$ co-occurs with word $j$ if $i$ is within 10 words to

\begin{tabular}{lrc}
\hline Representations & Accuracy & $95 \%$ CI \\
\hline Random & 62.00 & {$[61.28,62.53]$} \\
External GloVe & 72.19 & - \\
IMDB GloVE & 76.38 & {$[75.76,76.72]$} \\
Mittens & $\mathbf{7 7 . 3 9}$ & {$[77.23,77.50]$} \\
\hline
\end{tabular}

Table 2: IMDB test-set classification results. A difference of $1 \%$ corresponds to 250 examples. For all but 'External GloVE', we report means (with bootstrapped confidence intervals) over five runs of creating the embeddings and cross-validating the classifier's hyperparameters, mainly to help verify that the differences do not derive from variation in the representation learning phase.

the left or right of $j$, with the counts weighted by $1 / d$ where $d$ is the distance in words from $j$. Only words with at least 300 tokens are included in the matrix, yielding a vocabulary of 3,133 words.

For regular GloVe representations derived from the IMDB data - 'IMDB GloVE' - we train 50-dimensional representations and use the default parameters from Pennington et al. 2014: $\alpha=0.75, x_{\max }=100$, and a learning rate of 0.05. We optimize with AdaGrad (Duchi et al., 2011), also as in the original paper, training for $50 \mathrm{~K}$ epochs.

For Mittens, we begin with External GloVe. The few words in the IMDB vocabulary that are not in this GloVe vocabulary receive random initializations with a standard deviation that matches that of the GloVe representations. Informed by our simulations, we train representations with the Mittens weight $\mu=0.1$. The GloVe hyperparameters and optimization settings are as above. Extending the correlation analysis of fig. 1a to these real examples, we find that the GloVe representations generally have Pearson's $\rho \approx 0.37$, Mittens $\rho \approx$ 0.47 . We speculate that the improved correlation is due to the low-variance external GloVe embedding smoothing out noise from our co-occurrence matrix.

\subsection{IMDB Sentiment Classification}

The labeled part of the IMDB sentiment dataset defines a positive/negative classification problem with $25 \mathrm{~K}$ labeled reviews for training and $25 \mathrm{~K}$ for testing. We represent each review by the elementwise sum of the representation of each word in the review, and train a random forest model $(\mathrm{Ho}$, 1995; Breiman, 2001) on these representations. 
1. $\mathrm{No} / \mathrm{O}$ eye/R pain/R or/O eye/R discharge/R ./O

2. Asymptomatic/D bacteriuria/D ,/O could/O be/O neurogenic/C bladder/C disorder/C ./O

3. Small/C embolism/C in/C either/C lung/C cannot/O be/O excluded/O ./O

(a) Short disease diagnosis labeled examples. 'O': 'Other'; 'D': 'Positive Diagnosis'; 'C': 'Concern'; 'R': 'Ruled Out'.

Table 3: Disease diagnosis examples.
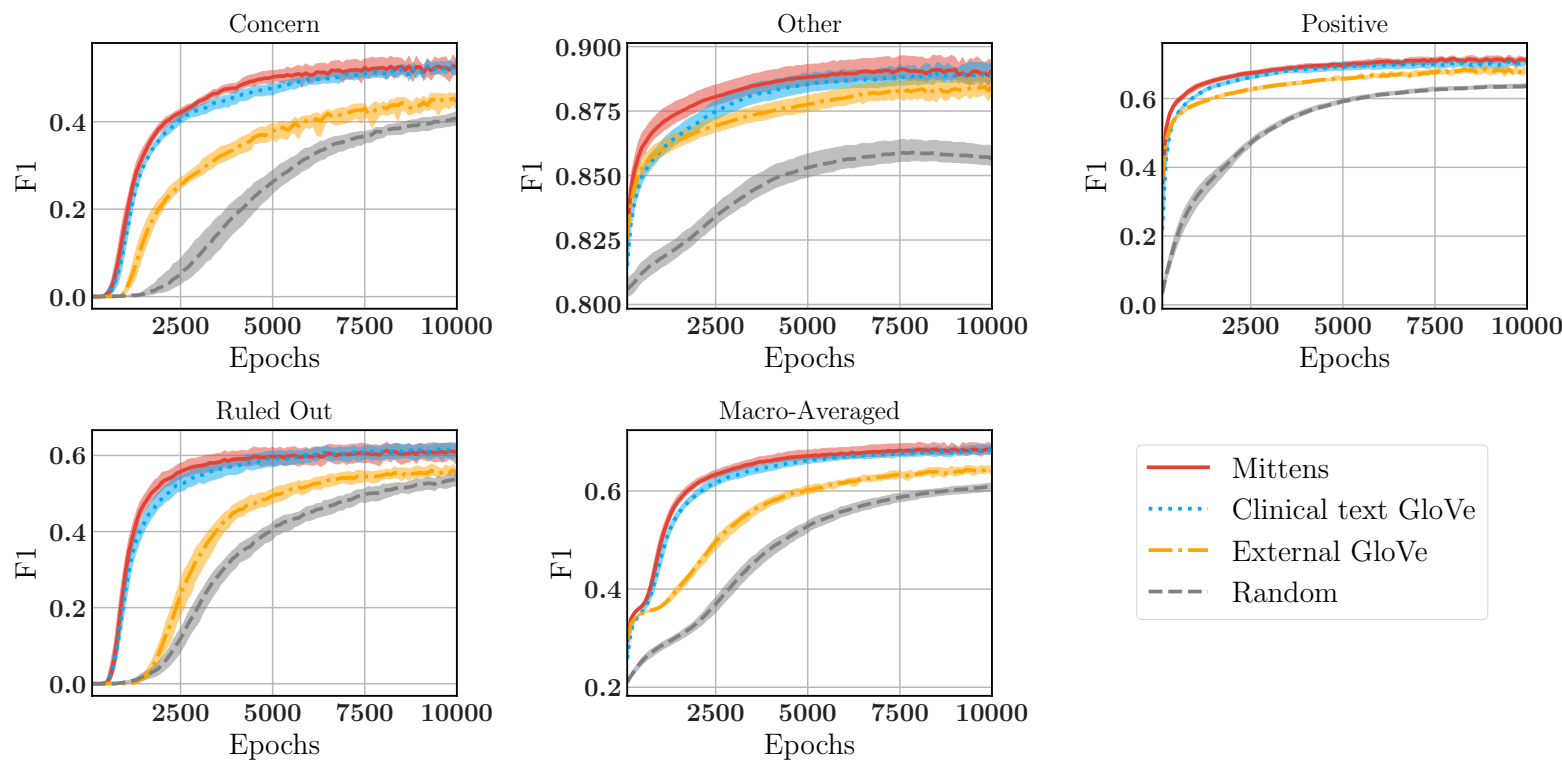

Figure 2: Disease diagnosis test-set accuracy as a function of training epoch, with bootstrapped confidence intervals. Mitten learns fastest for all categories.

The rationale behind this experimental set-up is that it fairly directly evaluates the vectors themselves; whereas the neural networks we evaluate next can update the representations, this model relies heavily on their initial values.

Via cross-validation on the training data, we optimize the number of trees, the number of features at each split, and the maximum depth of each tree. To help factor out variation in the representation learning step (Reimers and Gurevych, 2017), we report the average accuracies over five separate complete experimental runs.

Our results are given in tab. 2. Mittens outperforms External GloVe and IMDB GloVe, indicating that it effectively combines complementary information from both.

\section{Clinical Text Experiments}

Our clinical text experiments begin with $100 \mathrm{~K}$ clinical notes (transcriptions of the reports healthcare providers create summarizing their interactions with patients during appointments) from
Real Health Data. ${ }^{4}$ These notes are divided into informal segments that loosely follow the 'SOAP' convention for such reporting (Subjective, Objective, Assessment, Plan). The sample has 1.3 million such segments, and these segments provide our notion of 'document'.

\subsection{Word Representations}

The count matrix is created from the clinical text using the specifications described in sec. 3.1, but with the count threshold set to 500 to speed up optimization. The final matrix has a 6,519-word vocabulary. We train Mittens and GloVe as in sec. 3.1. The correlations in the sense of fig. 1a are $\rho \approx 0.51$ for both GloVe and Mittens.

\subsection{Disease Diagnosis Sequence Modeling}

Here we use a recurrent neural network (RNN) to evaluate our representations. We sampled 3,206 sentences from clinical texts (disjoint from the data used to learn word representations) containing disease mentions, and labeled these mentions as 'Positive diagnosis', 'Concern', 'Ruled Out', or

\footnotetext{
${ }^{4}$ http://www. realhealthdata.com
} 


\begin{tabular}{lrr}
\hline Subgraph & Nodes & Edges \\
\hline disorder & 72,551 & 408,411 \\
procedure & 53,616 & 264,000 \\
finding & 35,544 & 76,563 \\
organism & 33,721 & 41,090 \\
substance & 26,207 & 46,333 \\
\hline
\end{tabular}

(a) Subgraph sizes.

\begin{tabular}{lrrrrr}
\hline Representations & \multicolumn{6}{c}{ disorder procedure finding organism substance } \\
\hline Random & 56.05 & 55.97 & 75.14 & 68.15 & 64.72 \\
External GloVe & $\mathbf{6 9 . 3 1}$ & 65.89 & 80.72 & 74.12 & $\mathbf{7 7 . 5 8}$ \\
Clinical text GloVe & 66.19 & 64.96 & 79.18 & 73.42 & 73.37 \\
Mittens & 67.59 & $\mathbf{6 6 . 5 9}$ & $\mathbf{8 0 . 7 4}$ & $\mathbf{7 4 . 5 3}$ & 76.51 \\
\hline
\end{tabular}

(b) Mean macro-F1 by subgraph (averages from 10 random train/test splits). Italics mark systems for which $p \geq 0.05$ in a comparison with the top system numerically, according to a Wilcoxon signed-rank test.

Table 4: SNOMED subgraphs and results. For the 'disorder' graph (the largest), a difference of $0.1 \%$ corresponds to 408 examples. For the 'substance' graph (the smallest), it corresponds to 46 examples.

'Other'. Tab. 3a provides some examples. We treat this as a sequence labeling problem, using 'Other' for all unlabeled tokens. Our RNN has a single 50-dimensional hidden layer with LSTM cells (Hochreiter and Schmidhuber, 1997), and the inputs are updated during training.

Fig. 2 summarizes the results of these experiments based on 10 random train/test with $30 \%$ of the sentences allocated for testing. Since the inputs can be updated, we expect all the initialization schemes to converge to approximately the same performance eventually (though this seems not to be the case in practical terms for Random or External GloVE). However, Mittens learns fastest for all categories, reinforcing the notion that Mittens is a sensible default choice to leverage both domain-specific and large-scale data.

\subsection{SNOMED CT edge prediction}

Finally, we wished to see if Mittens representations would generalize beyond the specific dataset they were trained on. SNOMED CT is a public, widely-used graph of healthcare concepts and their relationships (Spackman et al., 1997). It contains $327 \mathrm{~K}$ nodes, classified into 169 semantic types, and 3.8M edges. Our clinical notes are more colloquial than SNOMED's node names and cover only some of its semantic spaces, but the Mittens representations should still be useful here.

For our experiments, we chose the five largest semantic types; tab. 4a lists these subgraphs along with their sizes. Our task is edge prediction: given a pair of nodes in a subgraph, the models predict whether there should be an edge between them. We sample $50 \%$ of the non-existent edges to create a balanced problem. Each node is represented by the sum of the vectors for the words in its primary name, and the classifier is trained on the concatenation of these two node representations. To help assess whether the input representations truly generalize to new cases, we ensure that the sets of nodes seen in training and testing are disjoint (which entails that the edge sets are disjoint as well), and we train on just $50 \%$ of the nodes. We report the results of ten random train/test splits.

The large scale of these problems prohibits the large hyperparameter search described in sec. 3.2, so we used the best settings from those experiments (500 trees per forest, square root of the total features at each split, no depth restrictions).

Our results are summarized in tab. $4 \mathrm{~b}$. Though the differences are small numerically, they are meaningful because of the large size of the graphs (tab. 4a). Overall, these results suggest that Mittens is at its best where there is a highlyspecialized dataset for learning representations, but that it is a safe choice even when seeking to transfer the representations to a new domain.

\section{Conclusion}

We introduced a simple retrofitting-like extension to the original GloVe model and showed that the resulting representations were effective in a number of tasks and models, provided a substantial (unsupervised) dataset in the same domain is available to tune the representations. The most natural next step would be to study similar extensions of other representation-learning models.

\section{Acknowledgements}

We thank Real Health Data for providing our clinical texts, Ben Bernstein, Andrew Maas, Devini Senaratna, and Kevin Reschke for valuable comments and discussion, and Grady Simon for making his Tensorflow implementation of GloVe available (Simon, 2017). 


\section{References}

Martín Abadi, Ashish Agarwal, Paul Barham, Eugene Brevdo, Zhifeng Chen, Craig Citro, Greg S. Corrado, Andy Davis, Jeffrey Dean, Matthieu Devin, Sanjay Ghemawat, Ian Goodfellow, Andrew Harp, Geoffrey Irving, Michael Isard, Yangqing Jia Jozefowicz, Rafal, Lukasz Kaiser, Manjunath Kudlur, Josh Levenberg, Dandelion Mané, Rajat Monga, Sherry Moore, Derek Murray, Chris Olah, Mike Schuster, Jonathon Shlens, Benoit Steiner, Ilya Sutskever, Kunal Talwar, Paul Tucker, Vincent Vanhoucke, Vijay Vasudevan, Fernanda Viégas, Oriol Vinyals, Pete Warden, Martin Wattenberg, Martin Wicke, Yuan Yu, and Zheng Xiaoqiang. 2015. TensorFlow: Large-scale machine learning on heterogeneous systems.

Piotr Bojanowski, Edouard Grave, Armand Joulin, and Tomas Mikolov. 2016. Enriching word vectors with subword information. ArXiv:1607.04606.

Leo Breiman. 2001. Random forests. Machine learning, 45(1):5-32.

Ignacio Cases, Minh-Thang Luong, and Christopher Potts. 2017. On the effective use of pretraining for natural language inference. ArXiv:1710.02076.

John Duchi, Elad Hazan, and Yoram Singer. 2011. Adaptive subgradient methods for online learning and stochastic optimization. Journal of Machine Learning Research, pages 2121-2159.

Dumitru Erhan, Yoshua Bengio, Aaron Courville, Pierre-Antoine Manzagol, Pascal Vincent, and Samy Bengio. 2010. Why does unsupervised pre-training help deep learning? The Journal of Machine Learning Research, 11:625-660.

Dumitru Erhan, Pierre-Antoine Manzagol, Yoshua Bengio, Samy Bengio, and Pascal Vincent. 2009. The difficulty of training deep architectures and the effect of unsupervised pre-training. In International Conference on Artificial Intelligence and Statistics, pages 153-160.

Manaal Faruqui, Jesse Dodge, Sujay Kumar Jauhar, Chris Dyer, Eduard Hovy, and Noah A. Smith. 2015. Retrofitting word vectors to semantic lexicons. In Proceedings of the 2015 Conference of the North American Chapter of the Association for Computational Linguistics: Human Language Technologies, pages 1606-1615, Stroudsburg, PA. Association for Computational Linguistics.

Tin Kam Ho. 1995. Random decision forests. In Proceedings of the Third International Conference on Document Analysis and Recognition, volume 1, pages 278-282. IEEE.

Sepp Hochreiter and Jürgen Schmidhuber. 1997. Long short-term memory. Neural Computation, 9(8):1735-1780.
Andrew L. Maas, Raymond E. Daly, Peter T. Pham, Dan Huang, Andrew Y. Ng, and Christopher Potts. 2011. Learning word vectors for sentiment analysis. In Proceedings of the 49th Annual Meeting of the Association for Computational Linguistics, pages 142150, Portland, Oregon. Association for Computational Linguistics.

Nikola Mrkšić, Diarmuid Ó Séaghdha, Blaise Thomson, Milica Gašić, Lina M. Rojas-Barahona, PeiHao Su, David Vandyke, Tsung-Hsien Wen, and Steve Young. 2016. Counter-fitting word vectors to linguistic constraints. In Proceedings of the 2016 Conference of the North American Chapter of the Association for Computational Linguistics: Human Language Technologies, pages 142-148. Association for Computational Linguistics.

Jeffrey Pennington, Richard Socher, and Christopher D. Manning. 2014. GloVe: Global vectors for word representation. In Proceedings of the 2014 Conference on Empirical Methods in Natural Language Processing (EMNLP), pages 1532-1543, Doha, Qatar. Association for Computational Linguistics.

Mohammad Taher Pilehvar and Nigel Collier. 2016. Improved semantic representation for domainspecific entities. In Proceedings of the 15th Workshop on Biomedical Natural Language Processing, pages 12-16. Association for Computational Linguistics.

Nils Reimers and Iryna Gurevych. 2017. Reporting score distributions makes a difference: Performance study of LSTM-networks for sequence tagging. arXiv:1707.09861.

Grady Simon. 2017. An implementation of GloVe in TensorFlow.

Kent A Spackman, Keith E Campbell, and Roger A Côté. 1997. SNOMED RT: A reference terminology for health care. In Proceedings of the AMIA Annual Fall Symposium, page 640. American Medical Informatics Association.

Stéfan van der Walt, S. Chris Colbert, and Gaël Varoquaux. 2011. The NumPy array: A structure for efficient numerical computation. Computing in Science and Engineering, 13:22-30.

Mo Yu and Mark Dredze. 2014. Improving lexical embeddings with semantic knowledge. In Proceedings of the 52nd Annual Meeting of the Association for Computational Linguistics (Volume 2: Short Papers), pages 545-550. Association for Computational Linguistics. 